\title{
Understanding intrinsic plasticizer in vegetable oil-based polyurethane elastomer as enhanced biomaterial
}

\begin{abstract}
Renewable polyol is of increasing interest as a building block in biomedical elastomer for bearing biodegradable ester group and immaculate functionality. Derived from non-edible vegetable oil, a new class of elastomer was successfully functionalized with MDI and TDI. Crosslink densities were varied by regulating ratio of hydroxyl to diisocyanate ( $\mathrm{r}$ ) at 1/1.0, $1 / 1.1$, and 1/1.2. Produced elastomers were examined by crosslink density, attenuated total reflectance Fourier transform infrared spectroscopy, differential scanning calorimetry, thermogravimetric analysis, dynamic mechanical analysis, tensile testing, and scanning electron microscopy. The obtained elastomers had subambient glass transition temperature ( $\mathrm{T}$ g) suggested majority soft segment that acted as a continuous phase with intermediate phase separation. Medium conversion at gel point had enhanced physical properties. Highly elastic mechanical behavior was afforded from combination of side chains and high molecular weight polyol. At $r=1 / 1.2$, MDI-based elastomer showed twofold improvement in Young modulus at slight expense of elongation. TDI-based elastomer accomplished elongation beyond $162 \%$. Branching allophanate and biuret resisted early thermal breakdown by elevating activation energy. Frequency response and kinetic of thermal degradation provided beneficial perspective for elastomer characterization. The vegetable oil-based polyurethane was found able to resemble most of the physical properties of polycaprolactone (PCL)derived polyurethane.
\end{abstract}

Keyword: Polyurethane elastomer; Biomaterial; Thermal properties; Degradation kinetic; Mechanical properties; Frequency response 